\title{
A New Enhanced Coordinated Multi-Point (CoMP) Transmission Design for Cancelling Inter-cell Interference for Cell-Edge Users Using Superimposed Supporting Signals
}

\author{
SHAHEER KHALID ${ }^{1}$, SADIQ IQBAL ${ }^{2}$, JEHAD M. HAMAMREH ${ }^{3}$ \\ ${ }^{1}$ WISLAB, Department of Electrical and Computer Engineering, Antalya Bilim University,Antalya, Turkey (e-mail: shaheer.khalid@ std.antalya.edu.tr) \\ ${ }^{2}$ WISLAB, Department of Electrical and Computer Engineering, Antalya Bilim University,Antalya, Turkey (e-mail: sadiq.iqbal@ std.antalya.edu.tr) \\ ${ }^{3}$ WISLAB, Department of Electrical and Electronic Engineering, Antalya Bilim University,Antalya, Turkey (e-mail:jehad.hamamreh@antalya.edu.tr ) \\ Corresponding author: Shaheer Khalid (e-mail: shaheer.khalid@std.antalya.edu.tr) Sadiq Iqbal (e-mail: sadiq.iqbal@std.antalya.edu.tr)
}

WISLAB (wislabi.com/solutions) offers solutions for building and deploying fully secure, cloud-based, and low-cost end-to-end 4G/5G networks along with providing consultations on helping companies reduce their networks CAPEX/OPEX cost and determine which solutions are best suited for their needs and use cases.

\begin{abstract}
Mobile data traffic is rapidly increasing with smartphones' massive spread and multimediadriven applications. To cope up with the data demand, operators will continue to deploy new small cells and update networks to advanced 4G LTE and leading-edge 5G NR technologies to take advantage of their superior capabilities. Despite their many improved features, the cell edge interference effect is still a very challenging problem in mobile wireless networks, and it has recently gained significant attention with the dawn of future generation mobile communications systems like $5 \mathrm{G}$ and $6 \mathrm{G}$ as such interference causes significant performance degradation in cell edge systems. Next-generation wireless communication systems will require higher spectral efficiency, enhanced system reliability, and much more improved SNR gain, which can be attained by the elimination of inter-cell interference, and more efficient utilization of resources than currently employed systems. Motivated by this observation, the proposed method in this article targets addressing the previously mentioned challenges and requirements, where we present a novel enhanced cooperative multiple points (eCoMP) technique that deploys two base stations to transmit superimposed user signals with the same spatial, temporal and spectral radio resources. In particular, we introduce and model the proposed concept of using supporting signals that are superimposed with the user signals to cancel inter-cell interference at the cell edge. This is achieved without sacrificing temporalspectral-spatial resources while providing low complexity at the receiver side. Computer simulations are conducted and analytical analysis is performed to verify the effectiveness and superiority of the proposed solution compared to other competitive schemes in the literature.
\end{abstract}

INDEX TERMS Coordination multi-point (CoMP), cell edge, multi-user, spectral efficiency, supporting signals, wireless communication, LTE advanced, 4G, 5G, 6G.

\section{INTRODUCTION}

$\mathbf{F}$ ifth-generation $(5 \mathrm{G})$ has introduced a paradigm shift in the wireless communication world. Instead of only focusing on increasing the data rates, $5 \mathrm{G}$ initialized diversifying the supported applications and use cases. $5 \mathrm{G}$ also catered to the enhancement of data rates under the enhanced mobile broadband (eMBB) service, expanded its vision to incorporate the increasing number of wireless devices with stringent reliability, low latency requirements under the massive machine type communication (mMTC), and ultra-reliable low latency communication (uRLLC) services, respectively [1]. However, even with the usage of two advanced transmission techniques like MIMO (MultipleInput Multiple-Output) and OFDM, which enhance the 
spectral efficiency of mobile-cellular networks and the spectral improvement at the cell border is still low. The nonuniform distribution of radio power in cellular networks is also the primary source of the cell edge problem [2]. In traditional cellular architecture, single or many antennas of the base station are collocated at the center of a cell. The received strength of a radio signal decreases exponentially with the propagation distance, causing substantial path loss for mobile terminals (MTs) near boundaries. The spectral performance at the cell edge is less than unity. The cell edge problem will become increasingly critical in the future [2].

Now, we will describe the techniques present in the literature that deal with inter-cell interference (ICI) and spectral efficiency at the cell-edges. The performance of multi-cell wireless networks severely limited by other-cell interference (OCI) due to co-channel transmission in other cells. This performance degradation is especially severe for users close to the cell edge. However, MIMO transmission theoretically provides a significant throughput gain, but the $\mathrm{OCI}$ is an obstacle due to the increased number of interfering sources [3].

ICI is also a well-known challenge for researchers from the beginning of mobile radio [4]. The techniques available in the literature focus on dealing with ICI but inter-cell interference coordination (ICIC) is a potential solution for dealing with the ICI mitigation issue in 3GPP LTE. The soft frequency reuse (SFR) and the fractional frequency reuse (FFR) schemes are two of the most extensively used ICIC techniques. Both SFR and FFR methods work by applying a frequency reuse factor of one in cell-center areas and a higher reuse factor in cell-edge locations. As a result, the ICI is low at the price of each cell's available frequency resources [5].

In [6], authors propose an ICI coordination technique based on users' ratio and frequency allocation, and this guarantees that cell-edge users in neighbor cells are not interfered with by each other but at the cost of BS resources. Authors in [7], propose an enhancement of MLD (Maximum Likelihood signal Detection) algorithm, with iterative signal detection with ICI cancellation based on an enhancement of MAP (Maximum A posteriori Probability) algorithm. However, this considerably increases overall system complexity. Authors in [8] introduce an effective signal detection method with ICI canceller for OFDM cellular systems. This algorithm also uses the MMSE QR decomposition of the virtual MIMO channel matrix and the M-algorithm to reduce the computational complexity of the original MLD. This scheme can improve the throughput performance of the MS at the cell edge in fully-loaded OFDM cellular systems. Yet, the cancellation of ICI and the improvement in throughput are achieved based on increased complexity.

The 3GPP launched LTE-Advance with coordination multi-point (CoMP) technology to tackle inter-cell interference [9]. CoMP network's principal goal is to allow geographically distant base stations (BSs) to work to- gether to serve the customer [10]. Coordinated Multipoint (CoMP) methods reduce interference through combining/coordinating transmission/scheduling schemes from various BSs, but at the expense of additional complexity and signaling overhead [11].

In [12], authors propose an ICI cancellation scheme by combining multi-cell coordinated scheduling and a simple linear interference canceling algorithm for the downlink transmission of MIMO/OFDM cellular systems, yet the use of scheduling increases latency in the system. Authors in the study [13] proposed an enhanced small cell grouping-based inter-cell interference control for dense small cell networks (SCN) that divided the small cells into different coordinated multipoint processing (CoMP) groups according to the mutual interference with adjacent small cells. They also propose a shifted frequency scheduling with dynamic power control to reduce inter-group interference. With this, the ICI can be controlled but not eliminated. In [14], the authors discuss the power division, reuse partitioning scheme with successive interference cancellation (PDICS) for OFDMA uplink transmission. PDICS balances ICI mitigation and spectral efficiency by appropriate frequency allocation and power distribution at the expense of network resources.

To tackle challenges such as ICI elimination and enhancing spectral efficiency at cell edges. A novel communication technique is needed that can meet the above-mentioned challenges without increasing the complexity and latency of the wireless network. Since the CoMP system meets the requirements related to ICI and spectrum efficiency, our case study is based on CoMP design.

The novelty and contributions of the proposed enhanced CoMP paradigm are as follows:

1) Efficient usage of the same radio and spatial resources for transmissions to all users at cell boundary with improved cell-edge throughput.

2) Specially designed superimposed supporting-signal $\left(\mathbf{a}_{1}, \mathbf{a}_{2}\right)$ base transmission for eliminating inter-cell interference.

3) Joint transmission is used to achieve multiplexing and diversity gain.

4) The proposed approach has low complexity because it employs superimposed support signals that automatically cancel inter-cell interference. As a result, reducing transceiver complexity.

5) Due to the channel matrices being diagonal, the inverse procedure is simple. As a result, the support signal matrices can be created using simple mathematical process.

In the subsequent sections, we will discuss the conventional CoMP techniques, overall system model, proposed algorithm, simulation results, and the conclusion.

\section{TECHNIQUES RELATED TO COMP}

In this section, a general description of CoMP techniques is presented that can be defined, in a much more straightforward manner as the coordination between transmission 


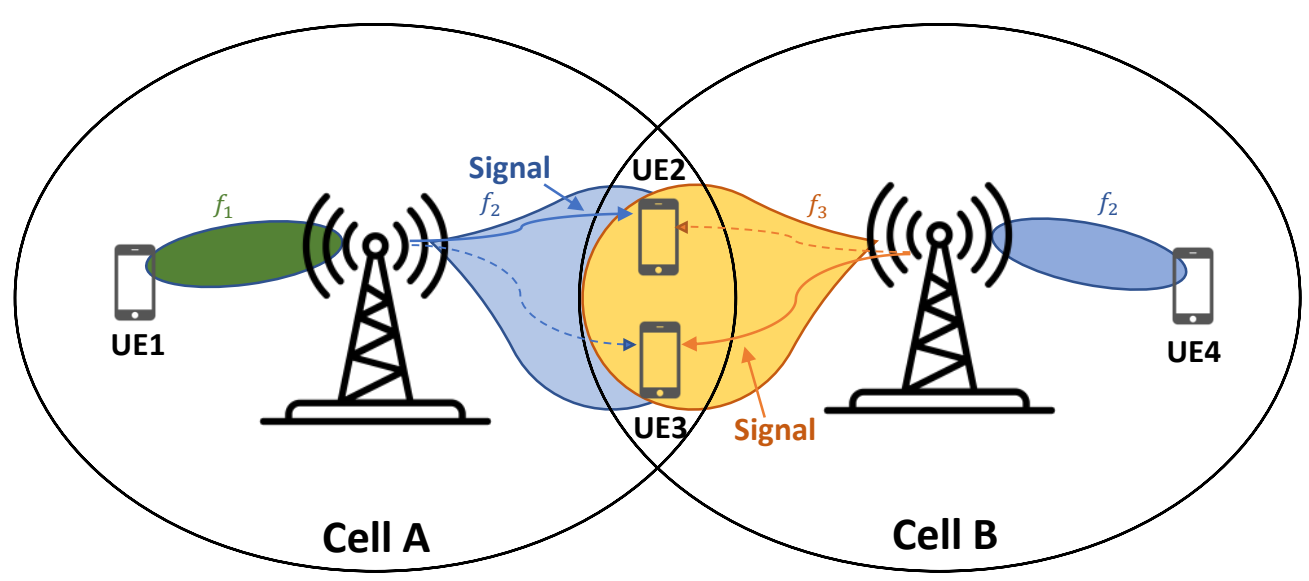

FIGURE 1. Coordinated Scheduling (CS).

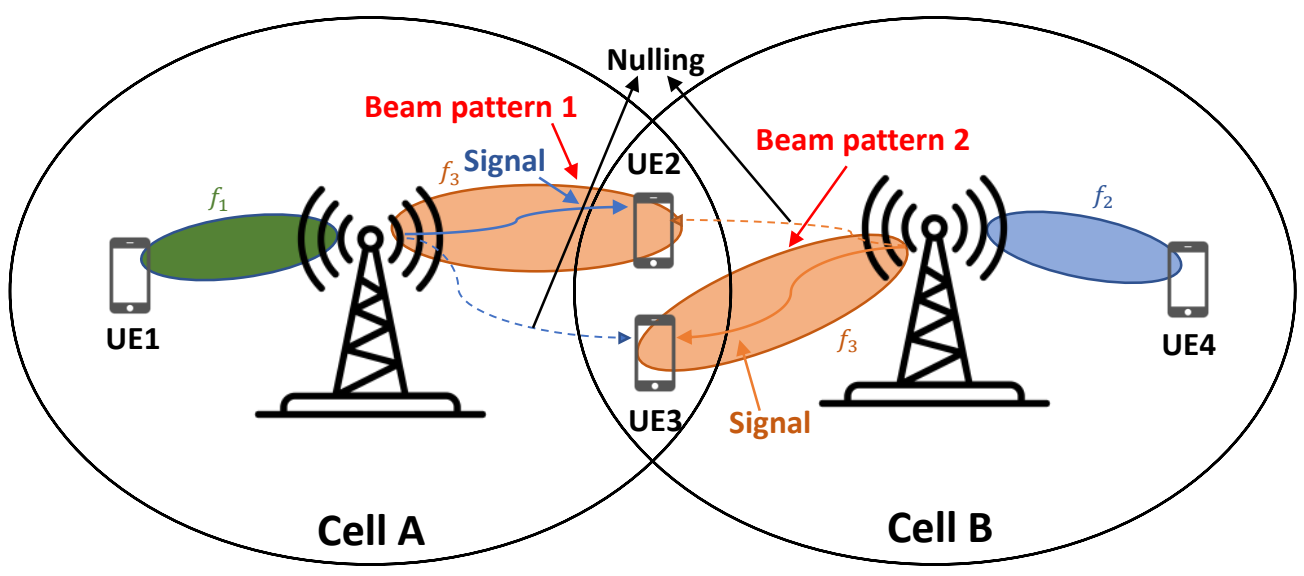

FIGURE 2. Coordinated Beamforming (CB).

points (TPs) and TPs generally termed as a set of collocated antennas distributed geographically [15]. Studies related to CoMP performed in 3GPP can generally be categorized as three different types of CoMP methods such as coordinated scheduling and coordinated beamforming (CS/CB), joint transmission (JT), and TP selection (TPS). In CS/CB, multiple coordinated TPs share only CSI for multiple UE terminals, while the data packets conveyed to a UE terminal are only available at one TP. JT is defined by the same data transmission from multiple coordinated TPs with the appropriate beamforming weights. TPS (or DPS), regarded as a special form of JT, where transmission of beamformed data for a UE terminal is performed at a single $\mathrm{TP}$ at each time instance, while the data is available at multiple coordinated TPs [15].

\section{A. COORDINATED SCHEDULING}

$\mathrm{CS}$ reduces inter-cell interference by allocating different frequency resources to cell-edge UEs. However, it requires a minimum operation time with complicated signal processing and an elaborate algorithm. In Fig. 1, UEs 2 and 3 at the cell 


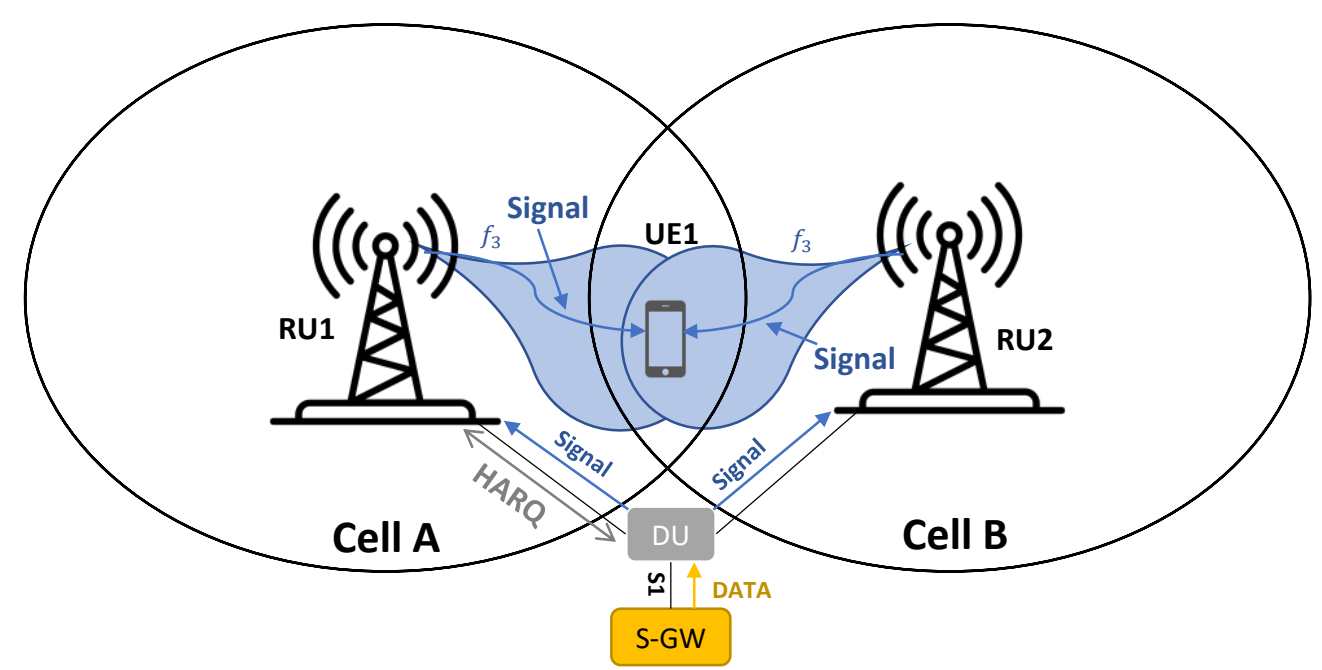

FIGURE 3. Joint Transmission (JT).

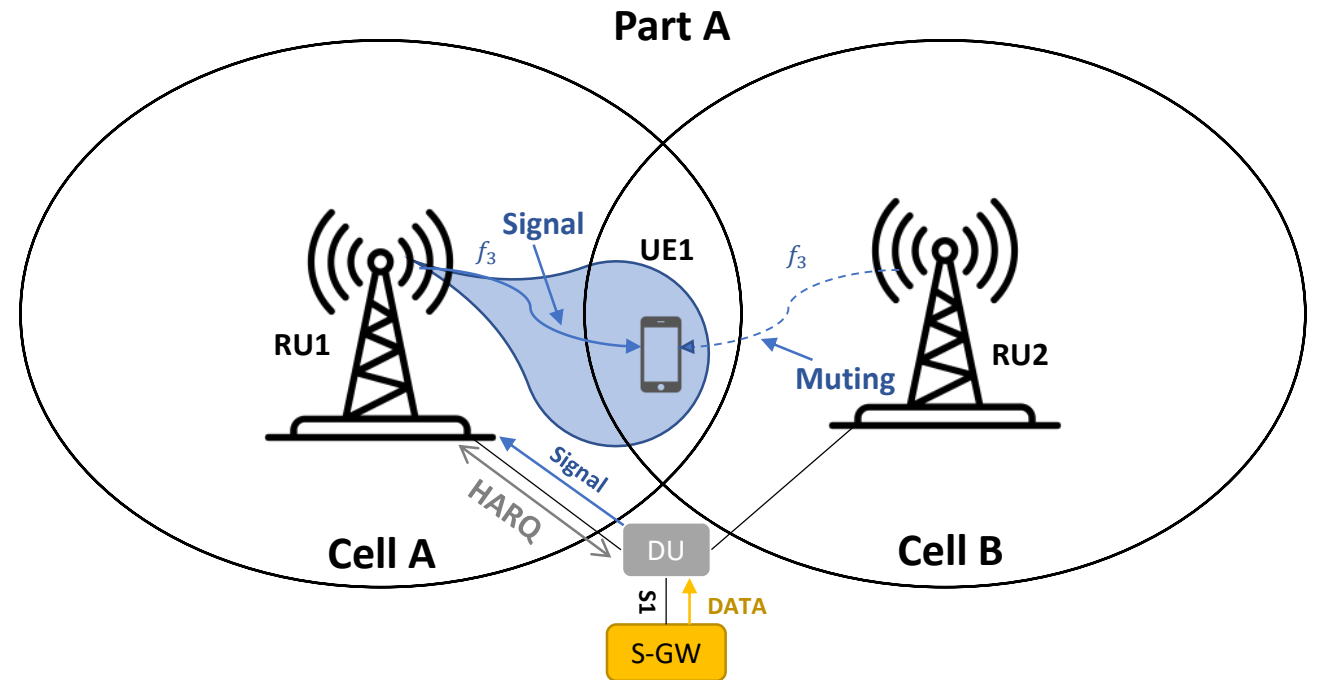

FIGURE 4. Dynamic Point Selection (DPS) Part A.

edges of cells A and B have different allocated frequency resources, can avoid interference, and improve throughput.

\section{B. COORDINATED BEAMFORMING}

$\mathrm{CB}$ can allocate different beam patterns to UEs at cell-edge by using intelligent antenna technology, which can allow cells A and B to cooperate and then prevent interference by directing the main beam to their UE and null-beam to the other neighbor UE as shown in Fig. 2.

\section{JOINT TRANSMISSION}

In JT, multiple cells transmit data simultaneously using the same radio resources, which leads to improved reception quality, yet there is no increase in data speed. In Fig. 3, the UE1 at the edge of cell A receives the same signal from both of the cells. This phenomenon enhances reception quality at the cell edge, thus improving the throughput. As shown in Fig. 3, HARQ is only performed at the serving BS while DU transmits data to RU1 and RU2. 


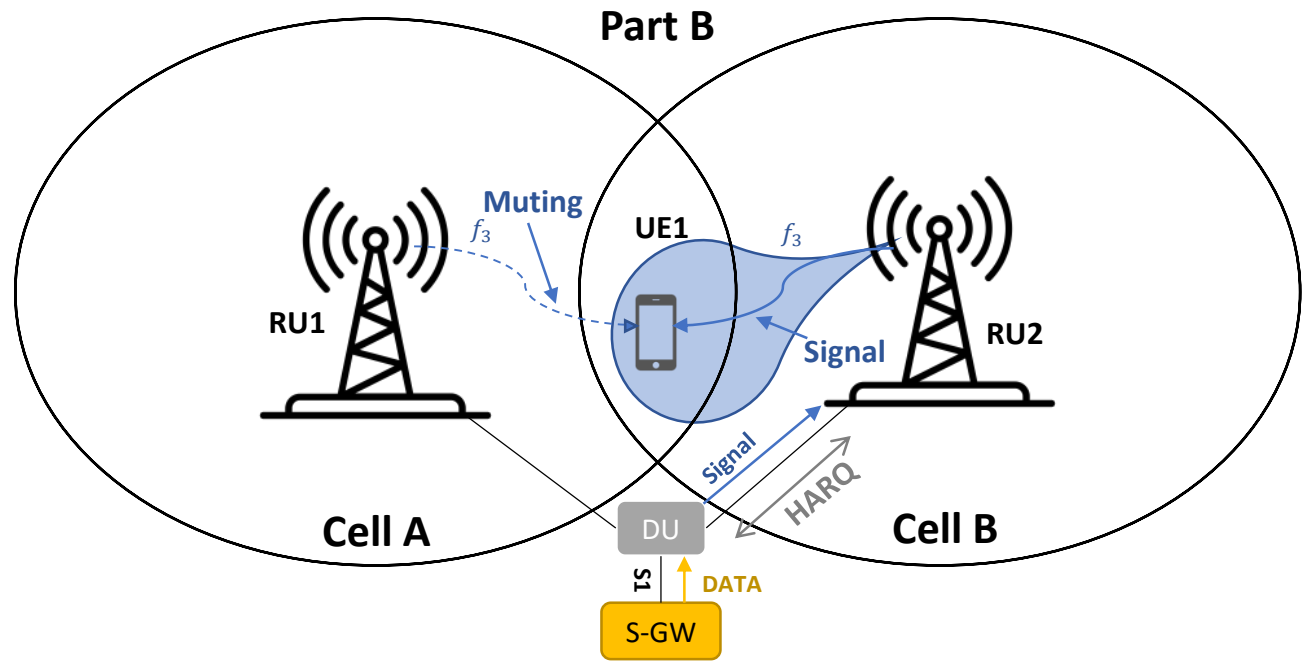

FIGURE 5. Dynamic Point Selection (DPS) Part B.

\section{DYNAMIC POINT SELECTION}

DPS operates exactly like JT in the preparation phase, where multiple cells share the same user data. However, during the data transmission phase, DPS functions differently by transmitting data through a cell (TP) with the lowest path loss and muting the other unselected cells, thus, improving reception quality at the user side since it receives the data from the cell that has better channel quality as shown in Figs. 4 and 5.

\section{OVERALL SYSTEM MODEL}

The proposed novel eCoMP technique may be scaled to accommodate any number of users. However, for simplicity purposes, the scheme mentioned in this work is designed for two users, whereas the system consists of two base stations BS1, and BS2, for serving two users. BS1 and BS2 are geographically separated and based in two different cooperative cells, each with a single antenna for transmission. As demonstrated in Fig. 6, the base stations in cells 1 and 2 transmit the signals that have the same user data for UE1 and UE2 superimposed with supporting signals. The UE1 and UE2 are present at the cell edges of both cells and attain coverage from both stations. The base stations adopt a joint transmission approach that improves users' reception quality by employing the same network resources like power, frequency, and beamforming, which leads to improved throughput at the cell edges. $\mathbf{H}_{\mathbf{k m}}$ is the diagonal channel frequency response of the user $k$ and base station $m$ during broadcasts [16]. The channels between BS1, UE1, BS2 UE2 are known at the transmitter and deemed to be slowly varying multi-path Rayleigh fading with the exponentially decaying channel.

Furthermore, we utilize channel-sounding techniques to determine the channels from the transmitter to the receiver. The proposed scheme allows the derivation of the wireless channels in a time division duplexing (TDD) system by determining the receiver to transmitter channels.

\section{PROPOSED ALGORITHMS}

This section illustrates the derivation of the mathematical model of the proposed technique. This work explores the use of supporting signals superimposed with the sum of user data to prevent inter-user interference, inter-cell interference at the cell edges, enhance throughput, improve reliability, and decrease complexity at the receiver. The proposed system employs joint transmission from two base stations, BS1, and BS2. During the transmission process, each BS transmits a signal that has one supporting signal $\left(\mathbf{a}_{1}\right.$ and $\left.\mathbf{a}_{2}\right)$, which is superimposed to the sum of the two users' data. The downlink transmission from the two separate base stations in two different cells ensures different channels, allowing for the design of supporting signals that provide reliable communication, no inter-cell interference, and efficient use of power.

The following is the design of the proposed algorithm. As illustrated in Fig. 7, the multi-carrier downlink system has two cells, each cell has one BS, and each BS has a single antenna employed. Furthermore, the system has two users, each with a single receive antenna as shown in Fig. 6. The two base stations transmit superimposed signals to users UE1 and UE2, located at the cell edge.

The total number of modulated symbols in one OFDM block at the transmitters for each user is $N_{f}$. As a result, each OFDM symbol's frequency response for UE1 and UE2 may be represented as $\mathbf{x}_{\mathbf{1}}=\left[\mathbf{x}_{\mathbf{0}}, \mathbf{x}_{\mathbf{1}}, \ldots, \mathbf{x}_{\mathbf{N}_{\mathbf{f}}-\mathbf{1}}\right]$ $\in C^{\left[N_{f} \times 1\right]}$ and $\mathbf{x}_{\mathbf{2}}=\left[\mathbf{x}_{\mathbf{0}}, \mathbf{x}_{\mathbf{1}}, \ldots, \mathbf{x}_{\mathbf{N}_{\mathbf{f}}-\mathbf{1}}\right] \in C^{\left[N_{f} \times 1\right]}$, re- 


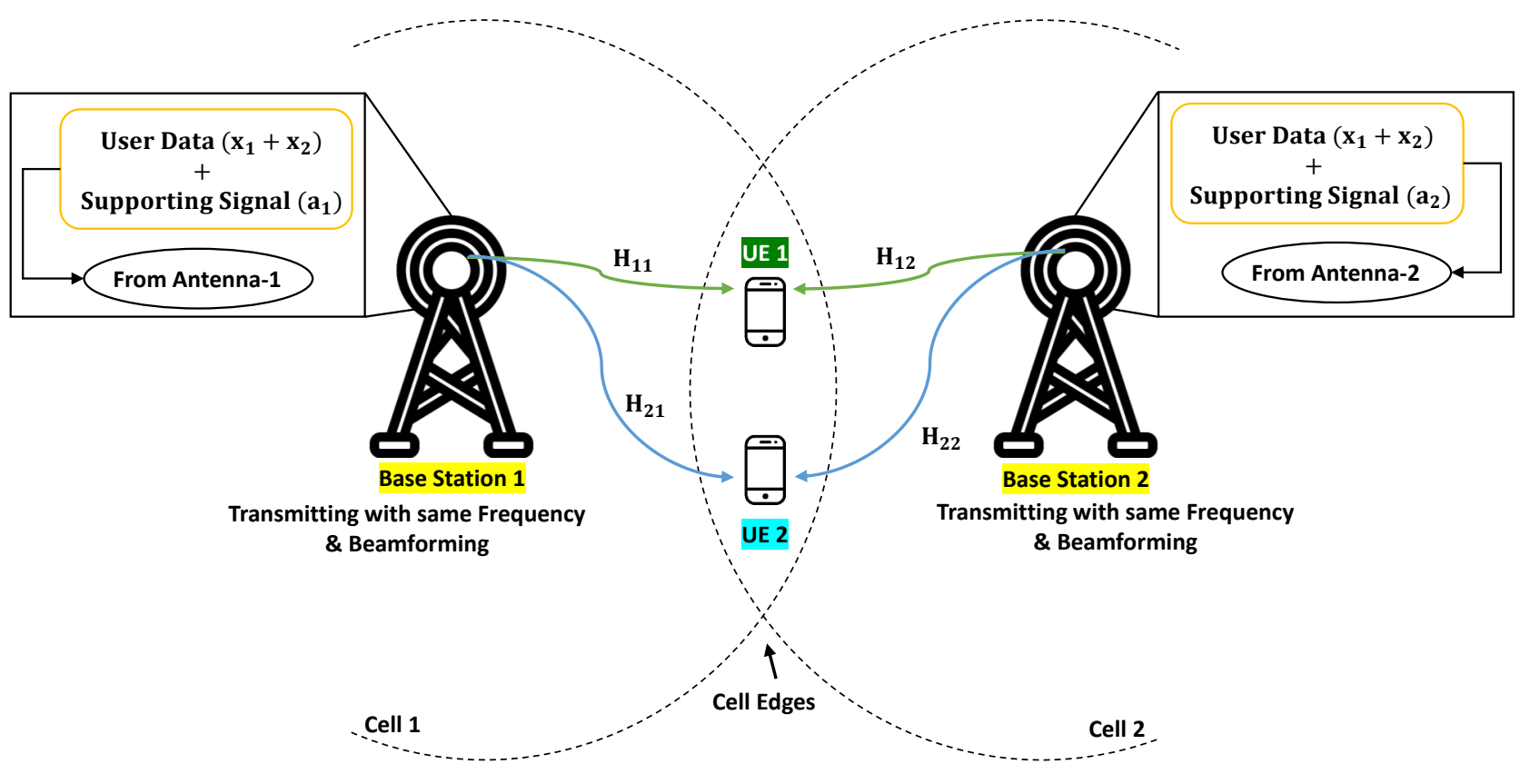

FIGURE 6. Detailed model of the proposed technique.

spectively. However, the supporting signals at both base stations are unique for each transmission. It should be noted that, $y_{k m} \in C^{\left[N_{f} \times 1\right]}, h_{k m} \in C^{\left[N_{f} \times N_{f}\right]}$, and $z_{k m} \in C^{\left[N_{f} \times 1\right]}$ represent the received signal, the diagonal matrix for channel frequency response, and the additive white gaussian noise (AWGN) between $k_{t h}$ user and active antenna $m_{t h}$ of the transmitter, respectively. After that, $\mathbf{x}_{\mathbf{1}}$ and $\mathbf{x}_{\mathbf{2}}$ are transformed from serial to parallel and added together. Furthermore, before transmission, the intended supporting matrices are added to the total sum of user symbols.

The superimposed transmitted signal from BS1 is represented as:

$$
\mathbf{u}_{1}=\mathbf{x}_{1}+\mathbf{x}_{\mathbf{2}}+\mathbf{a}_{1},
$$

Correspondingly, the transmitted superimposed signal from the BS2 may be represented as:

$$
\mathbf{u}_{2}=\mathbf{x}_{1}+\mathbf{x}_{2}+\mathbf{a}_{2},
$$

where $\mathrm{x}_{1}$ and $\mathrm{x}_{2}$ are frequency domain vector data designed for UE1 and UE2. Furthermore, $\mathbf{a}_{1}$ and $\mathbf{a}_{2}$ are the supporting matrices that are designed specially based on the legitimate receiver's channel. The $\mathbf{a}_{1}$ and $\mathbf{a}_{2}$ supporting signal will ensure that the UE1 and UE2 get robust signals that are free from interference even at the cell edges. The specifics of the received signals at UE1 and UE2 will be discussed in the subsequent subsections. Following that, we will explain the design of the supporting signals.

\section{A. RECEIVED SIGNAL AT USER-1 (UE1)}

The received signal at UE1 from transmission via BS1 can be described as:

$$
\mathbf{y}_{11}=\mathbf{H}_{11} \mathbf{u}_{1},
$$

where $\mathbf{H}_{11}$ represents the channel's frequency response between UE1 and BS1. Similarly, the received signal at UE1 from transmission via BS2 is represented as:

$$
\mathbf{y}_{12}=\mathbf{H}_{12} \mathbf{u}_{2},
$$

where $\mathbf{H}_{12}$ is the channel's frequency response between UE1 and BS2. The combined received signal at UE1 from transmission via BS1 and BS2 is given as follows:

$$
\hat{\mathbf{y}}_{1}=\mathrm{y}_{11}+\mathbf{y}_{12}+\mathbf{z}_{1},
$$

The combined received signal may be expressed after inserting the values of $\mathbf{y}_{11}$ and $\mathbf{y}_{12}$ in (5) as

$$
\hat{\mathbf{y}}_{1}=\mathbf{H}_{11} \mathbf{u}_{1}+\mathbf{H}_{12} \mathbf{u}_{2}+\mathbf{z}_{1},
$$

where $\mathbf{u}_{1}$ and $\mathbf{u}_{\mathbf{2}}$ are the superimposed transmitted signals and $\mathbf{z}_{1}$ is the additive white Gaussian noise (AWGN). The combined received signal is obtained by substituting the values of $\mathbf{u}_{1}$ and $\mathbf{u}_{2}$ in (6),

$$
\hat{\mathbf{y}}_{1}=\mathbf{H}_{11}\left(\mathrm{x}_{1}+\mathrm{x}_{2}+\mathbf{a}_{1}\right)+\mathbf{H}_{12}\left(\mathrm{x}_{1}+\mathrm{x}_{2}+\mathbf{a}_{2}\right)+\mathbf{z}_{1} .
$$




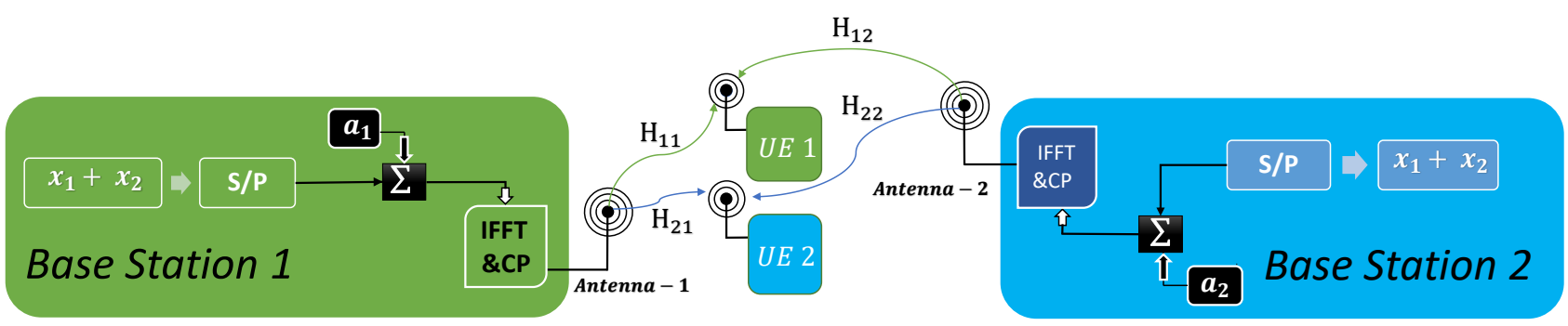

FIGURE 7. Basic Block Diagram of proposed eCoMP technique with support signal super positioning.

Rearranging (7) and combining like terms yields:

$$
\begin{gathered}
\hat{\mathbf{y}}_{1}=\left(\mathbf{H}_{11}+\mathbf{H}_{12}\right) \mathbf{x}_{1}+\left(\mathbf{H}_{11}+\mathbf{H}_{12}\right) \mathbf{x}_{2} \\
+\mathbf{H}_{11} \mathbf{a}_{1}+\mathbf{H}_{12} \mathbf{a}_{2}+\mathbf{z}_{1} .
\end{gathered}
$$

The first term in (8) is the desired term concerning UE1, whereas the remaining expressions are unwanted. The additional supporting signals will guarantee that unwanted terms, channel effects, and inter-cell interference are eliminated and canceled at UE1.

\section{B. RECEIVED SIGNAL AT USER-2 (UE2)}

The received signal at UE2 transmitted from BS1 is represented as in UE1:

$$
\mathbf{y}_{21}=\mathbf{H}_{21} \mathbf{u}_{1},
$$

where $\mathbf{H}_{21}$ is the channel's frequency response between UE2 and BS1. Similarly, the signal received at UE2 via $\mathrm{BS} 2$ is represented as:

$$
\mathbf{y}_{22}=\mathbf{H}_{22} \mathbf{u}_{2},
$$

where $\mathbf{H}_{\mathbf{2 2}}$ is the channel's frequency response between UE2 and BS2. The combined received signal at UE2 from transmissions done by BS1 and BS2 may be represented as

$$
\hat{\mathbf{y}}_{2}=\mathrm{y}_{21}+\mathrm{y}_{22}+\mathrm{z}_{2}
$$

where $\mathbf{y}_{\mathbf{2 1}}$ signifies the received signal at UE2 from BS1, and $\mathbf{y}_{\mathbf{2}}$ denotes the received signal at UE2 from BS2. After inserting the values of $\mathbf{y}_{21}$ and $\mathbf{y}_{22}$ into the equation (11), the combined received signal is stated as follows:

$$
\hat{\mathbf{y}}_{2}=\mathbf{H}_{21} \mathbf{u}_{1}+\mathbf{H}_{22} \mathbf{u}_{2}+\mathbf{z}_{2}
$$

The superimposed signals are represented by $\mathbf{u}_{\mathbf{1}}$ and $\mathbf{u}_{2}$. When the values of $\mathbf{u}_{1}$ and $\mathbf{u}_{2}$ are placed into the equation (12), the combined received signal indicated below is obtained.

$\hat{\mathbf{y}}_{2}=\mathbf{H}_{21}\left(\mathbf{x}_{1}+\mathbf{x}_{2}+\mathbf{a}_{1}\right)+\mathbf{H}_{22}\left(\mathbf{x}_{1}+\mathbf{x}_{2}+\mathbf{a}_{2}\right)+\mathbf{z}_{2}$.

Rearranging the equation (13) and combining similar terms yields:

$$
\begin{aligned}
\hat{\mathbf{y}}_{2}=( & \left.\mathbf{H}_{21}+\mathbf{H}_{22}\right) \mathbf{x}_{1}+\left(\mathbf{H}_{21}+\mathbf{H}_{22}\right) \mathbf{x}_{2} \\
& +\mathbf{H}_{21} \mathbf{a}_{1}+\mathbf{H}_{22} \mathbf{a}_{2}+\mathbf{z}_{2} .
\end{aligned}
$$

In the perspective of UE2, the second term in the equation (14) is the desired term, whereas the remaining components are undesirable. Likewise, the additional supporting signals will guarantee that undesirable terms, channel effects, and inter-cell interference are eliminated and canceled at UE2.

\section{DESIGNING THE SUPERIMPOSED SUPPORTING SIGNALS}

In this section, we will design the supporting signals $\mathbf{a}_{1}$ and $\mathbf{a}_{2}$ in such a way that the transmitted signals are received at the assigned receiver without the need for further calculations, resulting in interference-free, reliable, and much higher quality communication with less reception complexity. The supporting signals $\mathbf{a}_{\mathbf{1}}$ and $\mathbf{a}_{\mathbf{2}}$ are designed based on wireless channel characteristics. The design of supporting signals is motivated by the works in [17]-[19].

The desired term for UE1 is the first component of the equation (8). To calculate, the supporting signals $\mathbf{a}_{1}$ and $\mathbf{a}_{2}$ the second, third, and fourth term of the equation (8) should be equal to zero, as shown below:

$$
\left(\mathbf{H}_{11}+\mathbf{H}_{12}\right) \mathbf{x}_{2}+\mathbf{H}_{11} \mathbf{a}_{1}+\mathbf{H}_{12} \mathbf{a}_{2}=0 .
$$

Correspondingly, the second term of equation (14) is the desired term for UE2. As a result, the supporting signals $\mathbf{a}_{1}$ and $\mathbf{a}_{2}$ will be designed by equating the first, third, and fourth terms of equation (14) to zero: 


$$
\left(\mathbf{H}_{21}+\mathbf{H}_{22}\right) \mathbf{x}_{1}+\mathbf{H}_{21} \mathbf{a}_{1}+\mathbf{H}_{22} \mathbf{a}_{2}=0 .
$$

By jointly solving the equations (15) and (16), we get the values of supporting signals $\mathbf{a}_{1}$ and $\mathbf{a}_{2}$ as shown below.

$$
\begin{gathered}
\mathbf{a}_{2}=\frac{-\left(\mathbf{H}_{11}\right)\left(\mathbf{H}_{21}+\mathbf{H}_{22}\right) \mathbf{x}_{1}+\left(\mathbf{H}_{21}\right)\left(\mathbf{H}_{11}+\mathbf{H}_{12}\right) \mathbf{x}_{2}}{\mathbf{H}_{11} \mathbf{H}_{22}-\mathbf{H}_{12} \mathbf{H}_{21}} \\
\mathbf{a}_{1}=-\frac{\left(\mathbf{H}_{11}+\mathbf{H}_{12}\right) \mathbf{x}_{2}+\mathbf{H}_{12} \mathbf{a}_{2}}{\mathbf{H}_{11}}
\end{gathered}
$$

Using the supporting signals, $\mathbf{a}_{1}$ and $\mathbf{a}_{\mathbf{2}}$ shown in (17) and (18), signals for UE1 and UE2 are send from the two BSs as shown in Fig. 6. The supporting signals will guarantee complete reliability against channel effects and inter-cell interference with less complexity at the receiver side.

\section{SIMULATION RESULTS}

This section describes the simulation results for the proposed scheme to evaluate the effectiveness of the proposed strategy using performance metrics such as bit error rate (BER), packet error rate (PER), throughput error rate (TER), and peak to average power ratio (PAPR). The parameters used in the analysis are given in Table 1 below.

TABLE 1. System Parameters of the Proposed Algorithm

\begin{tabular}{ll}
\hline \hline Channel & Multipath Rayleigh Fading Channel \\
\hline Channel Length & 9 \\
\hline Cyclic Prefix (CP) & 9 \\
\hline FFT Size & 64 \\
\hline Modulation Type & BPSK \\
\hline \hline
\end{tabular}

We assume that the BS for each user employs OFDM with $N_{f}=64$ subcarriers and BPSK modulation, with a cyclic prefix (CP) of size $L$ to eliminate inter-symbol interference (ISI). As shown in Table 1, the channel between the transmitter and receiving nodes is a multi-path Rayleigh fading channel with an equal number of taps $L=9$.

Fig. 8 shows the BER versus SNR plots for the proposed method. As seen in Fig. 8, the BER outputs of UE1 and UE2 (depicted as proposed eCoMP-BER1 and proposed eCoMPBER2 respectively) are less than the conventional CoMP system, which means they perform well when compared to conventional CoMP and can remove inter-cell interference at cell edge users, indicating that the new technique can provide efficient and reliable communication due to (17) and (18).

Fig. 9 illustrates the throughput versus SNR plots for the proposed eCoMP method and conventional CoMP model. The graph displays the TER performances (proposed eCoMP-Throughput1 and proposed eCoMP-Throughput2 respectively) of UE1 and UE2 employing the proposed technique, the TER graphs prove the effectiveness of the proposed scheme, and this shows the throughput did not

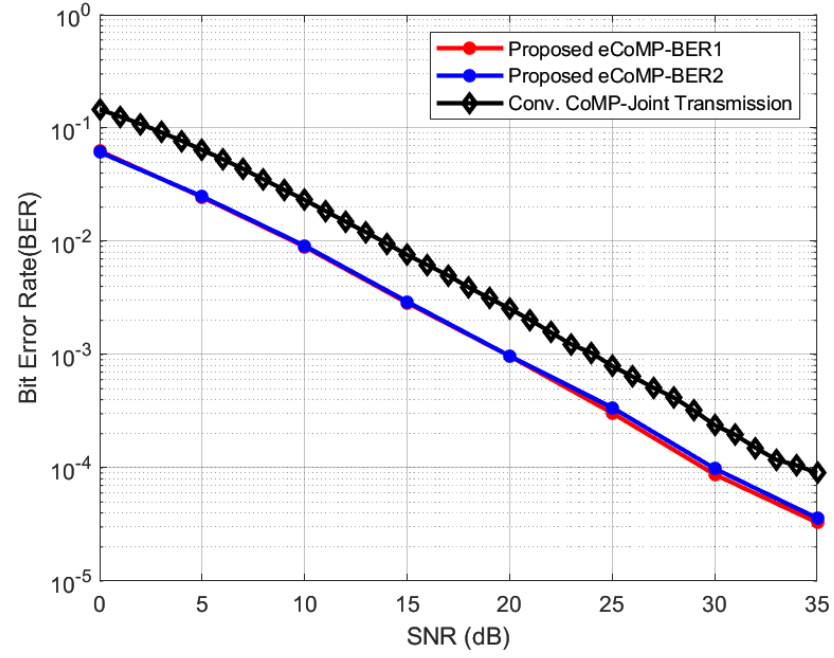

FIGURE 8. BER Vs SNR performance measure for the proposed algorithm

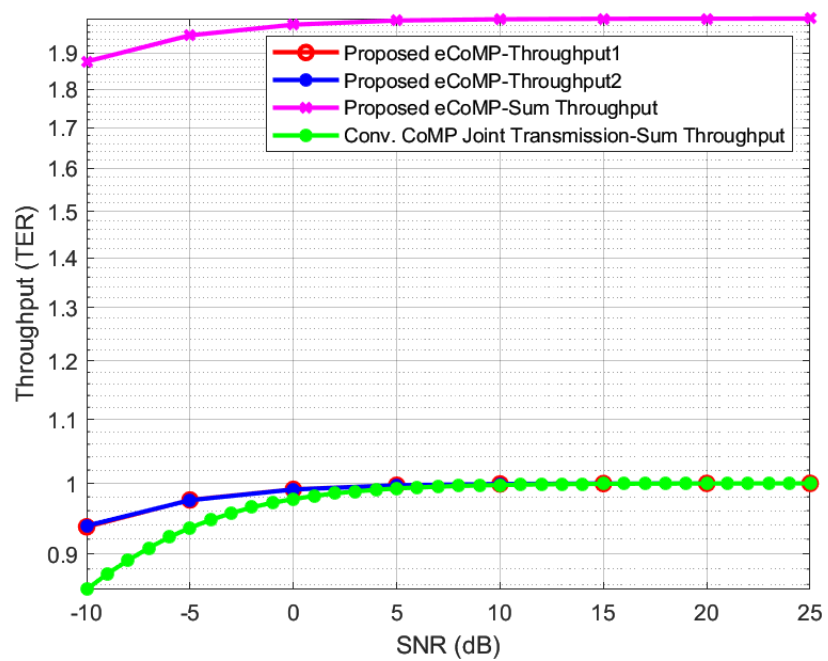

FIGURE 9. Throughput error rate of the proposed algorithm

decrease at the cell edges due to inter-cell interference after utilizing the proposed model. In Fig. 9, the sum throughput of the proposed model is illustrated, which is double the sum throughput of conventional CoMP while the conventional CoMP sum throughput is even less than the TER performances of both the UE1 and UE2. The comparison clearly describes the benefits of the proposed eCoMP model over conventional CoMP.

Fig. 10 represents the packet error rate (PER) of the proposed algorithm and illustrates the PER performances of UE1 and UE2 (proposed eCoMP-PER1 and proposed eCoMP-PER2 respectively). In Fig. 10, PER1 and PER2 performances demonstrate reliability and quality of service of the proposed eCoMP system, which is gained by utilizing superimposed supporting signals obtained by exploiting the channel's characteristics as specified in equations (17) and (18).

The peak-to-average-power-ratio (PAPR) graphs for BS1 


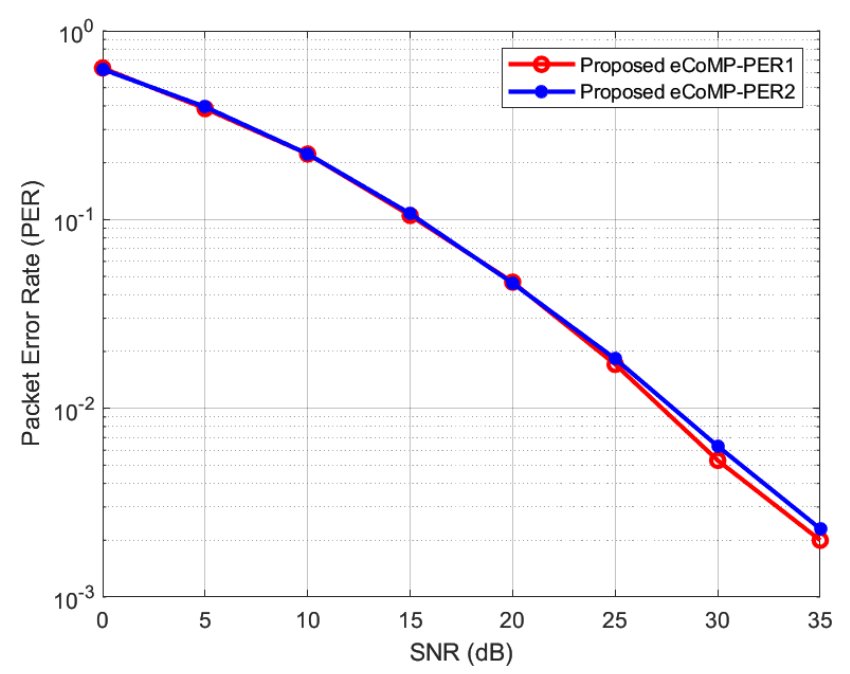

FIGURE 10. Packet error rate of the proposed algorithm.

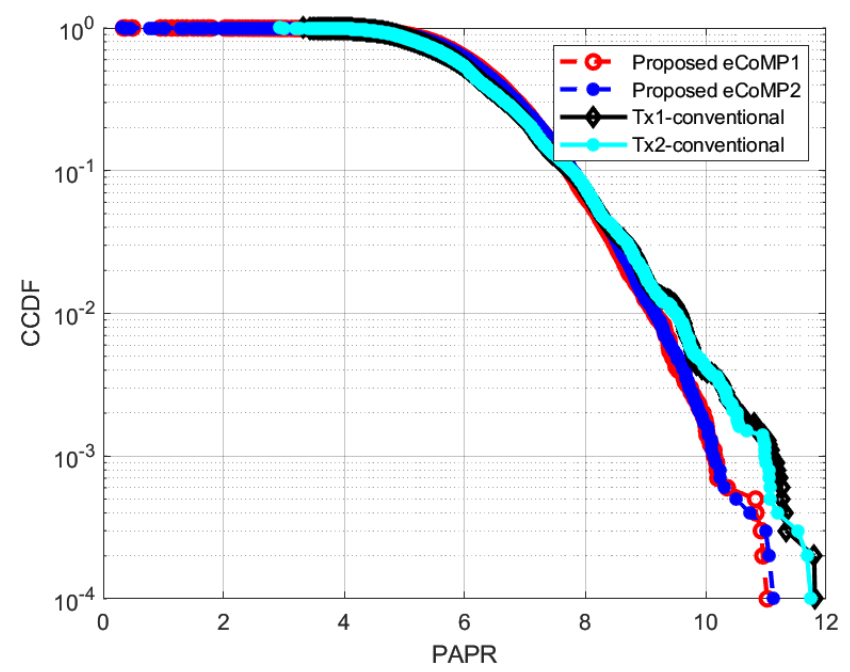

FIGURE 11. Peak to Average Power Ratio (PAPR) of the proposed algorithm.

(Proposed eCoMP1), BS2 (Proposed eCoMP1) and conventional OFDM are shown in the Fig. 11. At high SNR levels, the PAPR performance of the proposed system beats the traditional OFDM system, as shown in Fig. 11. The PAPR of the proposed method is less due to specially designed supporting signals. In conclusion, it tackles one of the most significant issues with the OFDM systems as in [20] by lowering the PAPR, which is highly desirable for low complexity devices and applications.

\section{CONCLUSION}

We propose a new eCoMP communication technique to eliminate inter-cell interference and improve throughput at the cell edges while conserving the spatial, temporal, and spectral radio resources. The proposed model consists of two cells with two base stations that have two users UE1, and UE2, at the boundaries of each cell. The superimposed transmit signals from BS1 and BS2 are designed based on users channels. Each transmission done has one superimposed supporting signal, guaranteeing that each user receives the signal intended for it. We verify and analyze the proposed paradigm with mathematical models and simulations. According to the observations, the proposed system provides users at the cell edges with ICI-free communication that has high throughput. When comparing the proposed model to conventional cell-edge interference reduction techniques, this technology utilizes the least power while providing better reliability with a low degree of complexity and efficient throughput at the cell edges. As a result, the suggested paradigm is well-suited to IoT applications that need little complexity and power. We intend to improve this communication technology in the future to allow for more than two users.

\section{REFERENCES}

[1] M. S. J. Solaija, H. Salman, A. B. Kihero, M. I. Sağlam, and H. Arslan, "Generalized coordinated multipoint framework for $5 \mathrm{~g}$ and beyond," IEEE Access, vol. 9, pp. 72 499-72 515, 2021.

[2] X. You, D. Wang, P. Zhu, and B. Sheng, "Cell edge performance of cellular mobile systems,' IEEE Journal on Selected Areas in Communications, vol. 29, no. 6, pp. 1139-1150, 2011.

[3] J. Zhang and J. G. Andrews, "Adaptive spatial intercell interference cancellation in multicell wireless networks," IEEE Journal on Selected Areas in Communications, vol. 28, no. 9, pp. 1455-1468, 2010.

[4] V. Jungnickel, K. Manolakis, W. Zirwas, B. Panzner, V. Braun, M. Lossow, M. Sternad, R. Apelfröjd, and T. Svensson, "The role of small cells, coordinated multipoint, and massive mimo in 5g," IEEE Communications Magazine, vol. 52, no. 5, pp. 44-51, 2014.

[5] J. Li, H. Zhang, X. Xu, X. Tao, T. Svensson, C. Botella, and B. Liu, "A novel frequency reuse scheme for coordinated multi-point transmission," in 2010 IEEE 71st Vehicular Technology Conference. IEEE, 2010, pp $1-5$.

[6] X. Fan, S. Chen, and X. Zhang, "An inter-cell interference coordination technique based on users' ratio and multi-level frequency allocations," in 2007 International Conference on Wireless Communications, Networking and Mobile Computing, 2007, pp. 799-802.

[7] M. Mikami and T. Fujii, "Iterative mimo signal detection with intercell interference cancellation for downlink transmission in coded ofdm cellular systems," in VTC Spring 2009 - IEEE 69th Vehicular Technology Conference, 2009, pp. 1-5.

[8] _ " A downlink transmission method for ofdm cellular systems with inter-cell interference cancellation using simplified mld based on mmse qrd-m algorithm," in VTC Spring 2008 - IEEE Vehicular Technology Conference, 2008, pp. 2011-2015.

[9] H. Xu and P. Ren, "Joint user scheduling and power control for celledge performance improvement in backhaul-constrained network mimo," in 2013 IEEE 24th Annual International Symposium on Personal, Indoor, and Mobile Radio Communications (PIMRC). IEEE, 2013, pp. 1342 1346.

[10] R. Irmer, H. Droste, P. Marsch, M. Grieger, G. Fettweis, S. Brueck, H.-P Mayer, L. Thiele, and V. Jungnickel, "Coordinated multipoint: Concepts, performance, and field trial results," IEEE Communications Magazine, vol. 49, no. 2, pp. 102-111, 2011.

[11] M. S. Ali, "On the evolution of coordinated multi-point (comp) transmission in lte-advanced," International Journal of Future Generation Communication and Networking, vol. 7, no. 4, pp. 91-102, 2014.

[12] M. Mikami and T. Fujii, "An inter-cell interference cancellation scheme with multi-cell coordinated scheduling for downlink of mimo/ofdm cellular systems," in 2011 IEEE 73rd Vehicular Technology Conference (VTC Spring), 2011, pp. 1-5.

[13] J. Huang, H. Zhang, W. Xu, and H. Zhang, "Grouping based inter-cell interference coordination in lte-a dense small-cell networks," in 2013 5th IEEE International Symposium on Microwave, Antenna, Propagation and EMC Technologies for Wireless Communications, 2013, pp. 78-83.

[14] L. Dong, Z. Song, L. Wenxin, and W. Wenbo, "A frequency reuse partitioning scheme with successive interference cancellation for ofdma uplink 
transmission," in 2009 IEEE 20th International Symposium on Personal, Indoor and Mobile Radio Communications, 2009, pp. 1362-1366.

[15] D. Lee, H. Seo, B. Clerckx, E. Hardouin, D. Mazzarese, S. Nagata, and K. Sayana, "Coordinated multipoint transmission and reception in Ite-advanced: deployment scenarios and operational challenges," IEEE Communications Magazine, vol. 50, no. 2, pp. 148-155, 2012.

[16] F. Z. Muhammad, H. M. Furqan, and J. M. Hamamreh, "Multi-cell, multi-user, and multi-carrier secure communication using non-orthogonal signals' superposition with dual-transmission for iot in $6 \mathrm{~g}$ and beyond," 2021.

[17] L. Joel, J. Hamamreh, and A. Mohamedou, "New non-orthogonal transmission schemes for achieving highly efficient, reliable, and secure multiuser communications," RS Open Journal on Innovative Communication Technologies, 2020 .

[18] J. Lemayian and J. Hamamreh, "A novel small-scale nonorthogonal communication technique using auxiliary signal superposition with enhanced security for future wireless networks," RS Open Journal on Innovative Communication Technologies, 102020.

[19] — - "Hybrid MIMO: A new transmission method for simultaneously achieving spatial multiplexing and diversity gains in MIMO systems," RS Open Journal on Innovative Communication Technologies, 52021.

[20] I. Baig, N. ul Hasan, M. Zghaibeh, I. U. Khan, and A. S. Saand, "A dst precoding based uplink noma scheme for papr reduction in $5 \mathrm{~g}$ wireless network," in 2017 7th International Conference on Modeling, Simulation, and Applied Optimization (ICMSAO). IEEE, 2017, pp. 1-4.

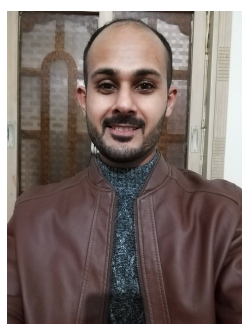

SADIQ IQBAL received the B.E. degree in electrical engineering from QUEST University Nawabshah, Pakistan in 2016. He is presently pursuing the master's (M.Sc.) degree in electrical and computer engineering. He is currently with Antalya Bilim University, Turkey.

His research interests include physical layer technology, 5G Communication networks, Artificial Intelligence, Deep Learning, and IoT and its applications..

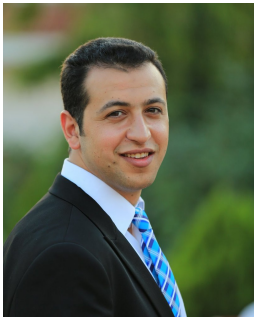

JEHAD M. HAMAMREH received the B.Sc. degree in electrical and telecommunication engineering from An-Najah University, Nablus, in 2013, and the Ph.D. degree in electricalelectronics engineering and cyber systems from Istanbul Medipol University, Turkey, in 2018. He was a Researcher with the Department of Electrical and Computer Engineering, Texas A and M University at Qatar. He is currently an Assistant Professor with the Electrical and Electronics Engineering Department, Antalya International (Bilim) University, Turkey.

His current research interests include wireless physical and MAC layers security, orthogonal frequency-division multiplexing multiple-input multiple-output systems, advanced waveforms design, multi-dimensional modulation techniques, IoT, 5G \& $6 \mathrm{G}$ and orthogonal/non-orthogonal multiple access schemes for future wireless systems. He is a Regular Reviewer for various refereed journals as well as a TPC Member for several international conferences.

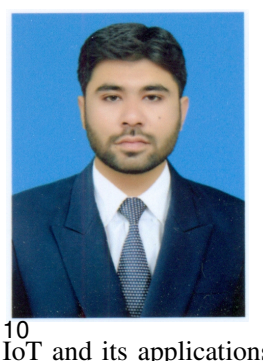

SHAHEER KHALID received a B.Sc. degree in electrical engineering from Government College University Faisalabad, Pakistan. He is presently pursuing a master's (M.Sc.) degree in electrical and computer engineering. He is currently with Antalya Bilim University, Turkey. He is currently working as a research assistant at Antalya Bilim University. His research interests include physical layer technology, 5G 6G Communication networks, Artificial Intelligence, Deep Learning, and 